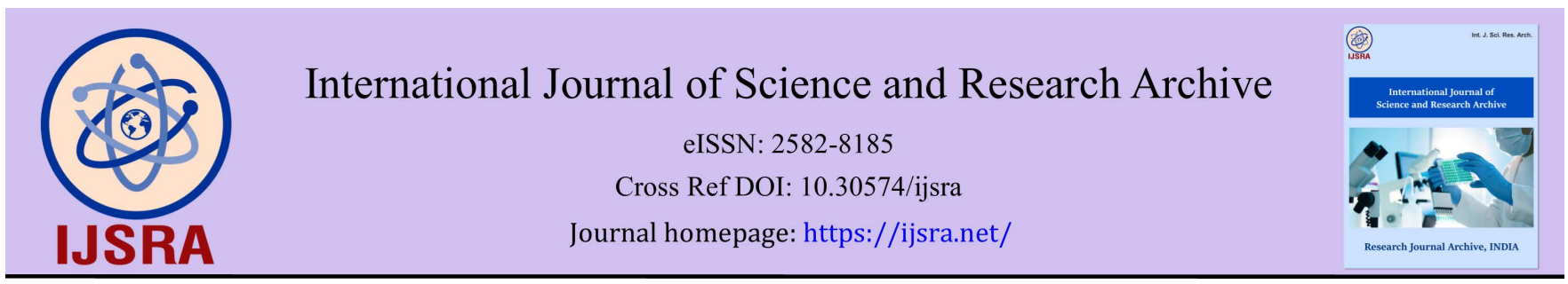

(RESEARCH ARTiClE)

Check for updates

\title{
Alcohol and Glucose D consumption decreases grip muscle strengths in graded doses
}

Omoirri Moses Aziakpono 1, *, Uyovwiesevwa Ataihire Johnson 2, Orji Uchechukwu Harrison 3, Chukwuemeka Charles Ofili ${ }^{2}$, Nwosu Gloria Chizoba ${ }^{4}$, Mba Ogbonnaya ${ }^{3}$, Olusola Ayobami John ${ }^{1}$ and Mbata Uchenna Chisom ${ }^{3}$

${ }^{1}$ Department of Pharmacology and Toxicology, Faculty of Pharmaceutical Sciences, Federal University of Oye-Ekiti, Ekiti State, Nigeria.

${ }^{2}$ Department of Public and Community Health, College of Medicine, Novena University Ogume, Delta State, Nigeria.

${ }^{3}$ Department of Pharmacology and Toxicology, Faculty of Pharmaceutical Sciences, Nnamdi Azikiwe University, Awka, Anambra State, Nigeria.

${ }^{4}$ Department of Pharmacy, Chukuemeka Odumegwu Ojukwu University Teaching Hospital, Amaku Awka, Anambra State, Nigeria.

International Journal of Science and Research Archive, 2021, 04(01), 039-046

Publication history: Received on 11 October 2021; revised on 20 November 2021; accepted on 22 November 2021

Article DOI: https://doi.org/10.30574/ijsra.2021.4.1.0176

\begin{abstract}
Alcohol, Blood Sugar, and Grip muscle strengths (GMS) may play key role in injury prevention and rehabilitation. In many cases, strengthening of the grip has been a prescription for rehabilitation from injuries such as golf and tennis elbow. In this study, the effects of alcohol and glucose consumption on grip muscle strength in early adult male subject was investigated. A total of thirty (200) human subjects of about18+ years old were recruited for the study. The subjects were administered varying doses of alcohol and glucose $\mathrm{D}$, then allowed to rest for a period of 5 and 10 minutes before assessing their grip muscle strength, fasting blood sugar (FBS) and body weights. Obtained records were then compared with those of control (normal) subject who neither received alcohol nor glucose D. Differences in mean was obtained between groups using the student t-test. From the result, study found a statistically significant decrease $(p<0.05)$ in GMS for different times ( $5 \mathrm{~min}$ and $10 \mathrm{~min}$ ) of alcohol administration as compared with control subjects. A notable significant decrease ( $p>0.05$ ) was also observed for FBS with increasing administration times as compared with control. This decrease was however insignificant for 10 min duration compared with 5 minutes. This decrease was however significant $(\mathrm{p}<0.05)$ for alcohol 5 min intake as compared with control group. All test group (experimental) showed a statistically significant decrease in GMS after alcohol administration as compared with control. Lastly, a statistically significant decrease $(\mathrm{p}<0.05)$ was observed in GMS for 10 min of Glucose D intake. Whereas, subject who took glucose D in 5 min showed an insignificant decrease as compared with control. It is recommended that more studies be conducted to corroborate the result of this study.
\end{abstract}

Keywords: Grip Strength; Glucose D; Alcohol; Blood Sugar

\section{Introduction}

The blood sugar concentration (or blood glucose level) is the amount of glucose in milligram (mg) present in one decilitre ( $\mathrm{dl}$ ) of the human blood it is measured in $\mathrm{mg} / \mathrm{dl}$ of blood, and referred to as the "fasting blood sugar" under fasting conditions $[1,2]$. As part of its homeostatic functions, the body tightly regulates this sugar levels, using hormonal agents as insulin or glucagon (both secreted in pancreas) [3]. If blood sugar levels increases or decreases than expected, counter-regulatory mechanisms are activated to stimulate glucagon release or inhibit it; depending on weather sugar is high or low [4]. For a healthy person (according to Guyton and Hall, 2006), the normal blood sugar level is some worth

\footnotetext{
${ }^{*}$ Corresponding author: Omoirri Moses Aziakpono

Department of Pharmacology and Toxicology, Faculty of Pharmaceutical Sciences, Federal University of Oye-Ekiti, Ekiti State, Nigeria.
}

Copyright $(2021$ Author(s) retain the copyright of this article. This article is published under the terms of the Creative Commons Attribution Liscense 4.0. 
between $65.0 \mathrm{mg} / \mathrm{dl}(3.6 \mathrm{mmol} / \mathrm{l})$ and $105.0 \mathrm{mg} / \mathrm{dl}(5.8 \mathrm{mmol} / \mathrm{l})$. During hunger, healthy people have a blood-glucose concentration of between 68 and $108 \mathrm{mg} / \mathrm{dl}$ (3.8 to $6.0 \mathrm{mmol} / \mathrm{l}$ ) in the morning [1].

Physiologically, while muscle fibres can exert a maximum force of approximately 30-40 newton for about the weight of a 3-4 kg per body mass adult, the strength of a muscle has been shown to be directly dependent on its size and cross sectional area. This in turn depends on dietetics. More so, a muscle's reflex time; which is "the elapsed time within which, neurochemicals are transduced for mechanical myo-contraction" will also be dependent on the availability of transmitter substances in the store during excitation. This factor is traceable to abundance of glucose and ATP in the muscle $[5,6]$

Though research involving supplements effectiveness during resistant exercise (RE) has been inconsistent, Carbohydrate-protein (CHO-PRO) and carbohydrate ( $\mathrm{CHO}$ ) beverages are two popular nutritional supplements currently used by amateur and professional athletes to improve exercise performance [7]. Ingesting a $\mathrm{CHO}$ and electrolyte beverage during exercise provides an immediate fuel source for working muscles and helps to offset body fluid losses from sweating to prevent dehydration [8]. Previous research has shown CHO supplementation to be effective for improving performance, attenuating perceived exertion, and reducing post-exercise cortisol levels during aerobic endurance exercises.

Available evidences suggest that CHO-PRO supplementation during aerobic endurance exercise can have similar or greater benefits on performance when compared to $\mathrm{CHO}$ supplementation alone $[9,10]$. Researchers have also shown that CHO-PRO supplementation can result in improved muscular adaptations, favourable hormonal responses, and enhanced recovery following resistance exercise (RE) compared to CHO supplementation [10]. Yet, it remains unclear whether CHO-PRO supplementation can improve performance, attenuate perceived exertion, reduce post-exercise, or even salivary cortisol responses to RE. This is of interest because attenuation of perceived exertion may allow an athlete to perform a greater volume of work due to reduced feelings of discomfort and fatigue. Furthermore, the reduction of post-exercise cortisol may improve the balance of muscle protein synthesis/degradation and aid in recovery [11].

In chronic hyperglycaemic conditions like Diabetes Mellitus (DM), sufferers, as part of its (DM) complications find it challenging to co-ordinate relaxes due to the extent to which nerve-muscle signal transduction has been affected [12]. This condition (diabetic neuropathy) clearly underscores the need for research into knowing the extent to which dietary may affect grip muscle strength; relative to glucose and alcohol intake to impact on nerve-muscle signalling and grip strength. Hence, the need for this study as well.

\section{Material and methods}

\subsection{Study Design}

The study adopted the cross sectional type of experimental research design. A total of two hundred (200) human subjects of about18+ years old were recruited from the student community of the aforementioned institution. The subjects were then administered variable doses of alcohol and glucose $\mathrm{D}$, then allowed to rest for a period of 5 and 10 minutes before assessing their grip muscle strength, fasting blood glucose and body weights. Obtained records were then compared with those of control subject who neither received alcohol nor glucose.

\subsubsection{Humans}

A total of two hundred (200) participants of 18+ years; comprising of adult males were recruited for the study.

\subsubsection{Glucometer}

An active Blood glucose monitoring device (Glucometer) with an Accu-Check trademark was purchased from Roche Diagnostics, Sandhofer Strasse 116, 68305 Mannheim, Germany (www.accu-check.com). Here is a list of the Ad-hoc accessories that accompanied the device;

- Glucose meter $\rightarrow$ the main Device

- Battery $\rightarrow$ of the CR2032 specification

- Test strip container $\rightarrow$ with accompanying 10 strips at the time of purchase

- A USB chord $\rightarrow$ for easy data synchronization with a PC

- A Bottle of Control solution $\rightarrow$ for servicing 
- A lancing device $\rightarrow$ for pricking the fingertip to obtain blood

- 10 pieces of lancet for the device

\subsubsection{Dynamometer}

A hand grip dynamometer (HGD) was obtained from the Department of Physiotherapy, Federal Medical Centre, Asaba, Delta State. The HGD had the following specifications:

- Capacity $\rightarrow 130 \mathrm{KG} / 286 \mathrm{LBS}$

- Brand Name $\rightarrow$ OEM

- Function $\rightarrow$ Arms

- $\quad$ Model Number $\rightarrow$ GYD4

- $\quad$ Type $\rightarrow$ location Hand Grips

- $\quad$ Product material $\rightarrow$ ABS plastic

- Product features $\rightarrow$ location

- Maximum Strength interval $\rightarrow$ 0-130KG

- Teaching Mode $\rightarrow$ Other

\subsection{Collection of Data}

\subsubsection{Estimating Alcohol Intake}

Alcohol intake (g/day) was assessed in two ways; (1) using a single 24-h daily recommended dose, an estimate of an individual's self-reported alcohol consumption on the day (2) by determining usual alcohol intakes with the NCI method, an estimate of long-term intake that employed data from 2 days of 24-h, via an alcohol intake questionnaire [13].

\subsubsection{Testing the Blood Sugar}

To obtain the fasting sugar value, a test strip was removed from its container. Thereafter, it was gently inserted into the test strip guide of the glucose meter (by sliding) until it was locked in place. This lead to the automatic turning on of the glucose meter, accompanied with a two second default display check. The code number on the label of the test strip container was then checked for a match with that of the default display, accompanied with a beep sound. This was necessary to be sure that the glucometer was on a ready-to-use mode. After cleaning and wash-drying with cotton wool (soaked in methylated spirit), an automated lancing device (with in-built lancing needle), was used to prick the subject's fingertip. The reason for cleansing with methylated spirit was to prevent possible infection at the site of puncture. Blood flow was then stimulated at puncture site, and a drop was applied to the centre of the green field of the glucometer's test strip. Applies blood smear was such that will be sufficient for detection by the glucose meter as stipulated in the manufacturer's guide. As soon as the blood smear was detected, a beep sound was produced and the fasting sugar level was shown on the display. This was immediately saved as read from the glucometer. Worth noting here is that, in most cases, no specific time was scheduled for this test, as long as participants had fasted for at least, 12 hours.

\subsubsection{Testing the Grip Muscle Strength (GMS)}

After a subject's fasting sugar status was measured (low, normal or high), a hand grip dynamometer was placed in their non-dominant hand. They were then made to stand in a position where the hand's grip muscles will rest in the direction of gravity. This gravitational-directional flow was a necessity for obtaining an optimum force from the limbs upon abduction beyond 90 degrees. Participants were then asked to squeeze the dynamometer with as much force as possible, being careful to squeeze only once for each measurement. Three trials were made with a pause of about 10-20 seconds between trials. This was necessary to avoiding the effects of muscle fatigue. In each case, the results were read, average taken, and recorded (in kilogram, $\mathrm{kg}$ ). Where the difference in scores was within $3 \mathrm{kgs}$, the test was accepted. However, where the difference between any two measures was more than $3 \mathrm{~kg}$, the test was repeated again. In the end, only the best 3 measurements (i.e. the highest three) were reported in the data sheet.

\subsubsection{Consent form}

Prior to determining participants' Blood pressures, informed consent was first sought in writing, haven orally briefed subjects on the points and usefulness of the study. A consent form was carefully structured in plain language, free from unnecessary words. It allowed participants to clearly respond to each of these points: 
- The participant were educated, haven read and understood information about the project

- The participant has been given the opportunity to ask questions

- The participant voluntarily agrees to participate in the project

- The participant understands that he or she can withdraw at any time without giving reasons and without penalty

- Procedures regarding confidentiality were explained (use of names, pseudonyms, anonymization of data, etc.)

- Use of the data in research, publications, sharing and archiving were explained

\subsubsection{Selection Criteria}

Selected subjects were within the age of 18+ years with no prior history of any cardiovascular disease like hypertension, cardiac arrhythmias, etc. subjects who also presented with catarrh or running nose were excluded from the study. Participants who were predisposed to occupational pollutants, were also excluded from the study.

\subsubsection{Ethical Consent}

Ethical consent was obtained from the Research and Ethics committee of the Faculty of Pharmaceutical Sciences, Nnamdi Azikiwe University, Awka, Anambra State. Also, before actual investigation, consent forms were administered to seek participants' permission.

\subsection{Statistical Analysis}

Results obtained from the study were expressed as Mean \pm SEM (Standard Error of Mean), while considering $p$ - value of less than $0.05(\mathrm{p}<0.05)$ to be statistically significant, a one-way analysis of variance (ANOVA) was used to determine the mean differences for variables between groups. Where a difference was found, the turkey (post hoc) test was performed to determine the source(s) of differences. All statistical procedure was performed with the graph pad prism (version 8.0).

\section{Results}

Figure 1 shows the various changes in grip muscle strength (GMS) for different durations of alcohol administration. From the figure, a statistically significant decrease $(\mathrm{p}<0.05)$ was observed in GMS for different times $(5$ min and 10 min) of alcohol administration as compared with control (not given alcohol) subjects

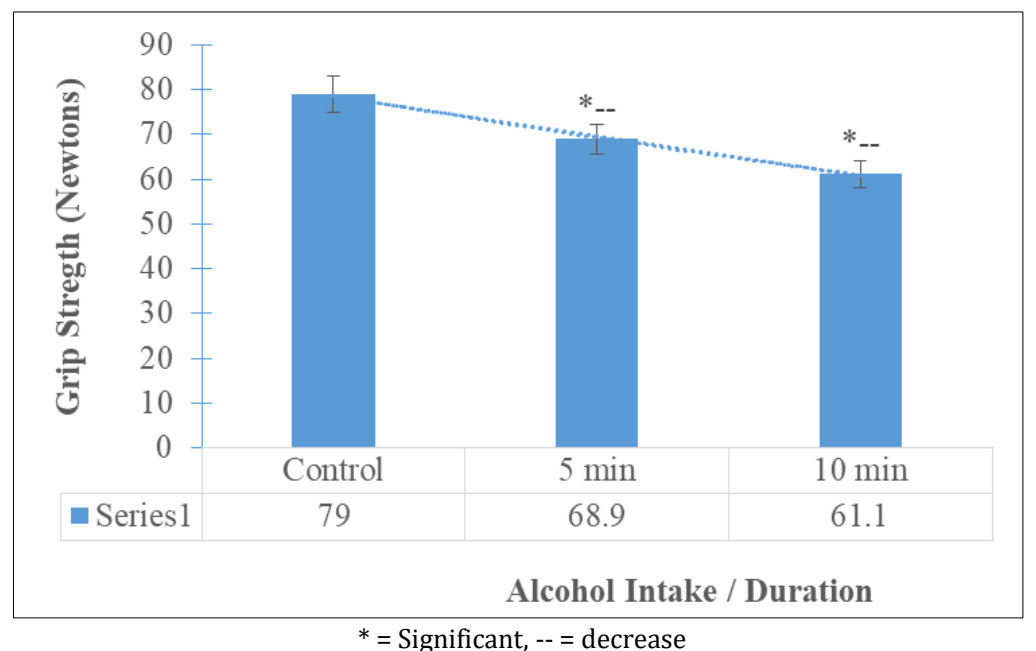

Figure 1 Comparative Changes in Grip Muscle Strength at Different Durations of Alcohol Intake

chat (Figure 2) shows the comparative effects of alcohol intake (at different durations of 5 and 10 minutes) on the fasting blood sugar (FBS) levels. From the chat, a noticeable significant decrease $(p>0.05)$ was observed for FBS with increasing administration times as compared with control. This decrease was however insignificant for 10 min duration compared with 5 minutes 
The comparative changes in grip muscle strengths (GMS) before and after alcohol intake is shown in above figure (figure 3). From the figure, all test group (experimental) showed a statistically significant decrease in GMS after alcohol administration as compared with control.

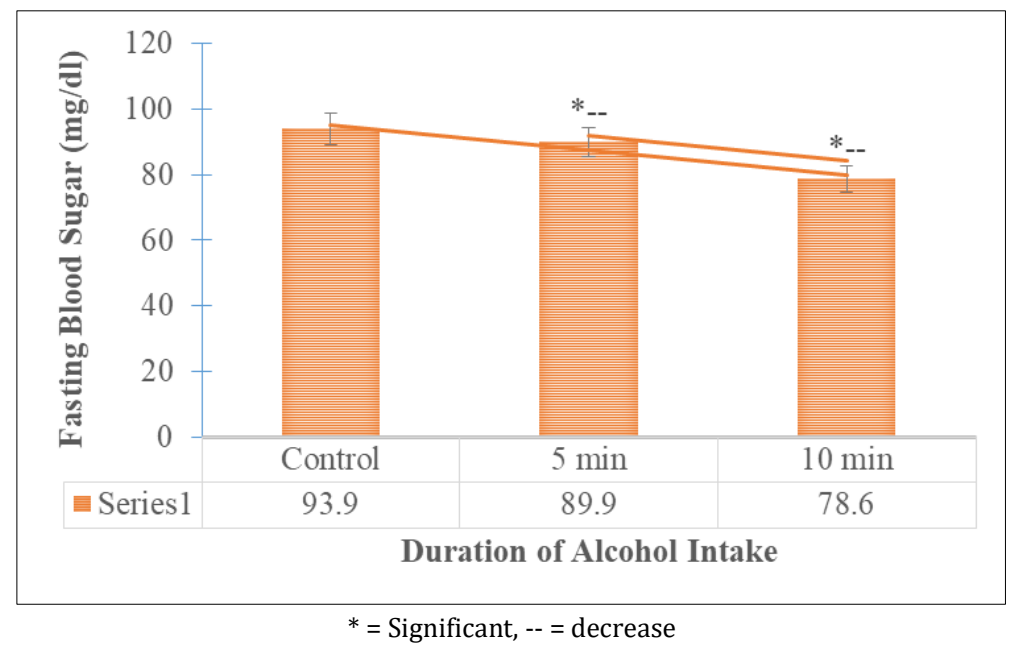

Figure 2 Comparative Effects of Alcohol Intake on Fasting Blood Sugar

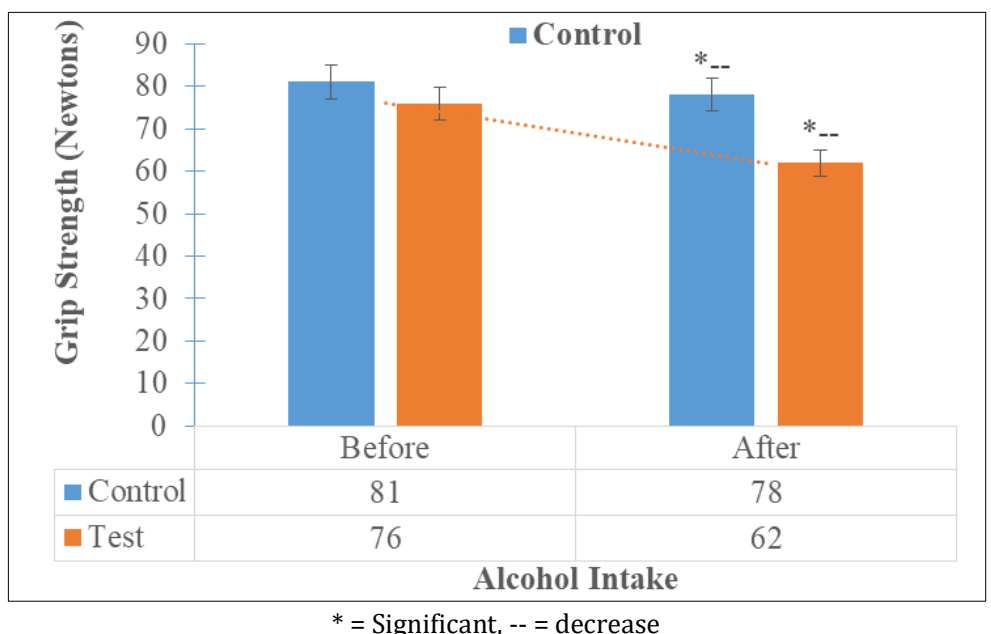

Figure 3 Comparative Changes in Grip Muscle Strengths before and after Alcohol Intake

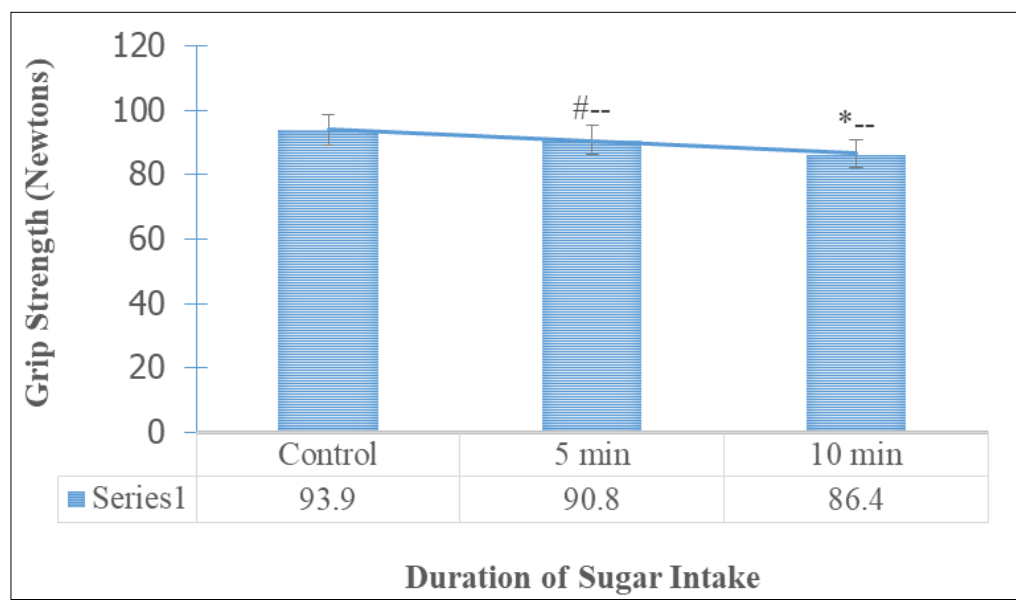

* = Significant, \# = Insignificant, -- = decrease

Figure 4 Comparative Changes in Grip Muscle Strengths for durational Glucose-D Intake 
The comparative changes in grip muscle strengths (GMS) for varying durations of glucose D intake is shown in above figure (figure 4). From the figure, a statistically significant decrease ( $<<0.05)$ was observed in GMS for 10 min of Glucose D intake. Whereas, subject who took glucose D in 5 min showed an insignificant decrease as compared with control.

\section{Discussion}

There is a growing research database which suggests that skeletal muscles, particularly postural muscles of the lower limbs undergo atrophy, structural and metabolic alterations during space flight. What appears inconclusive however is the effect that varying blood sugar levels has on gross muscular efficiency, as well as grip muscle strengths (GMS). In this study, the effects of varying doses of alcohol and glucose-D intake on GMS was investigated. A total of two hundred (200) human subjects of about18+ years old were ethically recruited from the student community of the Nnamdi Azikiwe University, Awka, Anambra State. The subjects were then administered variable doses of alcohol and glucose $\mathrm{D}$, then allowed to rest for a period of 5 and 10 minutes before assessing their grip muscle strength, fasting blood glucose and body weights. Obtained records were then compared with those of control subject who neither received alcohol nor glucose.

From figure I, the various changes in GMS for different durations of alcohol and glucose-D administration is reported. From the figure, a statistically significant decrease $(\mathrm{p}<0.05)$ was observed in GMS for different time durations $(5$ min and $10 \mathrm{~min}$ ) of alcohol administration as compared with control (not given alcohol) subjects. This effect shows that alcohol intake may decrease GMS in humans, especially with increasing duration of administration time.

In a slightly similar report, Smith et al (2006) found a direct correlation in grip strength and overall body strength in old females. Their study revealed that, "grip strength was moderately correlated with overall body strength in the very old and oldest populations [14]. Though in theory, one would believe the two are correlated and more studies may be necessary for other population.

Many of the research studies correlated grip strength to various other physical variables including nutritional status, rotator cuff weakness, fatigue, and overall physical function. In his book "Science of Sports Training", sport scientist Thomas Kurz recommended the measurement of handgrip strength using a hydraulic dynamometer to reveal the physical readiness of an athlete. This information provides valuable data to the coach with regards to an athlete's training status. If an athlete's grip strength is percentage kilograms below baseline or previous workout, the athlete may be fatigued. If the opposite is true, the athlete will have recovered optimally and performance may increase. This theory draws parallel to the findings of studies performed by Hunt, Rowlands and Johnston, 1985. Each of these studies used handgrip dynamometric testing to evaluate physical functioning in surgical, lifestyle disease, and mid to late life subjects. Findings from each of the studies correlated less than optimal physical functioning or fatigue with lower strength scores in handgrip dynamometric testing. Again from figures 1 and 2, a comparative change in grip muscle strength at different durations of alcohol intake, as well as a comparative effect of alcohol intake on fasting blood sugar are respectively shown. Specifically, figure 2 shows the comparative effects of alcohol intake (at different durations of 5 and 10 minutes) on the fasting blood sugar (FBS) levels.

From the chat, a noticeable significant decrease ( $p>0.05$ ) was observed for FBS with increasing administration times as compared with control. This decrease was however insignificant for 10 min duration compared with 5 minutes. Contrarily, the various changes in grip muscle strength for different durations of alcohol administration revealed a statistically significant decrease ( $p<0.05$ ) in GMS for different times (5 min and $10 \mathrm{~min}$ ) of alcohol administration as compared with control (not given alcohol) subjects. The implication of this finding is that, even though slight variations exist, alcohol and glucose-D intakes work in opposite fashion to decrease and increase the human GMS respectively at any given instance in time.

In a similar investigation in 2005 on "Carbohydrate supplementation and perceived exertion during muscular exercise", Utter et al., (2005) found No significant difference between some randomly selected thirty strength-trained subjects who were subjected to weightlifting for 2 hours (4 sets with 10 maximum repetitions; 10 exercises, with 2-3-minute rest intervals) $[15,16]$. After the ingestion of $10 \mathrm{ml} / \mathrm{kg}$ of $6 \%$ carbohydrate or placebo beverages during a resistancetraining bout, They came up with the finding that Carbohydrate supplements exerted no attenuating effect on ratings of perceived exertion (RPE) during resistance training. In a similar vein, Nieman et al., (2006) found a significantly higher Plasma Glucose, insulin, and ferric reducing ability with a significantly lower isoprostanes, cortisol, and epinephrine in carbohydrate versus placebo group of Fifteen subjects who functioned as their own controls by ingesting carbohydrate (6\%) or placebo in a randomized design while cycling for 2.5 hours (approximately $75 \%$ V.0 (2 peak)) [17]. Even though the decrease in muscle glycogen was not different during cycling exercise, they however claimed that carbohydrate ingestion and increased stress hormones may apparently have influence on muscular oxidative stress. 
A few studies have shown no improvement in performance from CHO-PRO compared to CHO supplementations. Whereas others have seen improvements from CHO-PRO supplementation performance; Investigators have demonstrated that the addition of protein to a carbohydrate supplement during exercise may have additional benefits not observed in CHO supplementation alone. It is believed that the plasma insulin response increases in an effort to reduce significant decreases in muscle glycogen that occur during high volume RE.

Additionally, evidence has shown that branched-chain amino acids (leucine, isoleucine, and valine) found in most protein supplements, can attenuate perceived exertion and reduce mental fatigue during exercise by influencing the synthesis, concentration, and release of neurotransmitters in the brain. As a result, the use of a CHO-PRO supplement may improve performance and attenuate perceived exertion during RE to a greater extent when compared to a CHO only supplement. Moderate intensity, high volume RE has been shown to elicit acute anabolic and catabolic hormonal responses in the body.

Acute increases in anabolic hormones such as insulin and testosterone can enhance protein synthesis in skeletal muscle and promote muscle growth. Conversely, increases in catabolic hormones such as cortisol attempt to provide energy in response to declines in muscle glycogen and blood glucose by stimulating gluconeogenesis and peripheral release of substrates. Cortisol stimulates protein degradation and decreases protein synthesis in muscle cells resulting in increased amino acid availability in the circulation. This is further converted to energy via gluconeogenesis [16]. Investigators have shown that performing RE without supplementation will result in a significant post-exercise increase in salivary cortisol; indicative of an increased muscle protein breakdown. While a few studies have shown a reduced post-exercise plasma cortisol response to RE due to CHO-PRO supplementation, it appears that the post-exercise salivary cortisol response to RE due to CHO-PRO supplementation has not been examined [2]. Additionally, cortisol is a physiological marker for stress and some studies suggest it may be a mediator of perceived exertion during exercise [16]. Cortisol typically increases during the later portion of prolonged exercise and is correlated with higher ratings of perceived exertion. In 1999, Utter et al. found that higher levels of plasma glucose due to carbohydrate supplementation were associated with lower levels of cortisol and attenuated ratings of perceived exertion during prolonged cycling. Therefore, it is also of interest to examine the effects of CHO-PRO and CHO supplementation on the post-exercise salivary cortisol response to RE.

\section{Conclusion}

This study has shown using humans that, a perfect relationship exists between fasting blood sugar, grip muscle strength, alcohol and glucose intake. Apparently, increasing oral dose of glucose-D increased the grip muscle strength, whereas in hypoglycaemic males and females show increase and decrease in FBS respectively. Alcohol consumption on the other hand decreased muscle performance at increasing dose administration. Thus, under clinical and laboratory settings, it may be possible to access a subject's blood sugar range and grip muscle strength with the goal of setting precisions before proper prognosis.

\section{Compliance with ethical standards}

\section{Disclosure of conflict of interest}

Authors declare that no conflict of interest of any sort exists in this work

\section{Statement of informed consent}

Informed consent was obtained from all individual participants included in the study.

\section{References}

[1] Guyton AC, Hall JE. Release of Energy from the Glucose Molecule by the Glycolytic Pathway; Textbook of Medical Physiology. Elsevier, philadephia. 2006; 11: 832-839.

[2] Baty JJ, Hwang H, Ding Z, Bernard JR, Wang B, Kwon B. Effect of a carbohydrate and protein supplement on resistance exercise performance, hormonal response, and muscle damage. J Strength \& Conditioning Res. 2007; 21(2): 321-329.

[3] Bird SP, Tarpenning KM, Marino FE. Effects of liquid carbohydrate / essential amino acid ingestion on acute hormonal response during a single bout of resistance `exercise in untrained men. Nutrition. 2006; 22: 367-375. 
[4] Bird SP, Tarpenning KM, Marino FE. Independent and combined effects of liquid carbohydrate/essential amino acid ingestion on hormonal and muscular adaptations following resistance training in untrained men. European J Applied Physiol. 2006; 97: 225-238.

[5] Haff GG, Koch AJ, Potteiger JA, Kuphal KE, Magee LM, Green SB. Carbohydrate supplementation attenuates muscle glycogen loss during acute bouts of resistance exercise. J Sport Nutrition \& Exercise Metabolism. 2006; 10(3): 326-339.

[6] Haff GG, Lehmkuhl MJ, McCoy LB, Stone MH. Carbohydrate Supplementation and Resistance Training. J Strength \& Conditioning Res. 2003; 17(1): 187-196.

[7] Ivy JL, Res PT, Sprague RC, Widzer MO. Effect of a carbohydrate-protein supplement on endurance performance during exercise of varying intensity. J Sport Nutrition \& Exercise Metabolism. 2003; 13: 382-395.

[8] Nova E, Baccan GC, Veses A, Zapatera B, Marcos A. Potential health benefits of moderate alcohol consumption: current perspectives in research. Proc Nutr Soc. 2012; 71: 307-15.

[9] Ramano-Ely BC, Todd MK, Saunders MJ, Laurent TS. Effect of an isocaloric carbohydrate-protein antioxidant drink on cycling performance edicine and Science in Sports and Exercise. 2006; 38(9): 1608-1616.

[10] Rasmussen BB, Tipton KD, Miller SL, Wolf SE, Wolfe RR. An oral essential amino acid-carbohydrate supplement enhances muscle protein anabolism after resistance exercise. J Applied Physiol. 2000; 88: 386-392.

[11] Saunders MJ, Kane MD, Todd MK. Effects of carbohydrate-protein beverage on cycling endurance and muscle damage. Medicine and Science in Sports and Exercise. 2004; 36(7): 1233-1238.

[12] OM Odigie, AO Naiho, EK Nwangwa, EM Aisuodione, JC Igweh. Correlation of Human Fasting Blood Sugar with Grip Muscle Strength and Reflex Response Time. Journal of Advances in Medicine and Medical Research. 2016; 14(6): 1-10.

[13] Nova E, Baccan GC, Veses A, Zapatera B, Marcos A. Potential health benefits of moderate alcohol consumption: current perspectives in research. Proc Nutr Soc. 2012; 71: 307-15.

[14] Smith T, Smith S, Martin M, Henry R, Weeks S, Bryant A. Grip Strength in Relation to Overall Strength and Functional Capacity in Very Old and Oldest Old Females. The Haworth Press Inc. 2006; 63-78.

[15] Utter AC, Kang J, Neiman DC, Brown VA, Dumke CL, McAnulty SR. Carbohydrate supplementation and perceived exertion during resistance exercise. J Strength \& Conditioning Res. 2015; 19(4): 939-943.

[16] Utter AC, Kang J, Nieman DC, Dumke CL, McAnulty SR, McAnulty LS. Carbohydrate attenuates perceived exertion during intermittent exercise and recovery. Medicine and Science in Sports and Exercise. 2007; 39(5): 880-885.

[17] Nieman DC, Henson DA, Gojanovich G, Davis JM, Murphy EA, Mayer EP, Pearce S, Dumke CL, Utter AC, McAnulty SR, McAnulty LS. Res Sports Med. 2006; 14(3): 225-237.

[18] Williams AG, Ismail AN, Sharma A, Jones DA. Effects of resistance exercise volume and nutritional supplementation on anabolic and catabolic hormones. European J Applied Physiol. 2002; 86: 315-321. 